of Behring, material is developed in the blood which neutralizes the effects of the specific poison of the disease, and confers immunity to those in whom the serum of such blood is artificially introduced. The studies of Pfeiffer have, however, led him to the conclusion that substances are formed which destroy the infectious material and therefore eradicate the cause of the disease. It may be that cell action is so stimulated and the metabolic processes so changed that effectual resistance is offered, through the medium of the blood serum, to the further invasion by the infectious material. This is probable, as experimenters have found that the supposed pathogenic microörganisms may be cultivated in the blood serum of animals immunized for the disease which they produce. Moreover, antitoxins are held in solution in the blood, but do not pass through the coats of the vessels.

\section{SERUM THERAPY IN DIPHTHERIA.}

\author{
Read in the Section on Materia Medica, Pharmacy and Therapeu- \\ ics at the Forty-seventh Annual Meeting of the Americen \\ BY EDWIN ROSENTHAL, M.D. \\ PHILA DELPHIA, PA.
}

Since the introduction of the diphtheria antitoxin as a specific remedy in diphtheria, $I$ have used it in 127 cases of diphtheria with a record of five deaths.

As the character of my cases were in many instances of the most dangerous variety, and in nineteen instances necessitated the operation of intubation, I may not be misjudged if I ascribe to the specific action of antitoxin the results obtained.

To briefly summarize my work they are: Tonsillar 67; pharyngeal and tonsillar 14; nasal, pharyngeal and tonsillar 1 ; pharyngeal 1; laryngeal 12; tonsillar and laryngeal 18; pharyngeal, tonsillar and laryngeal 8; nasal, pharyngeal, tonsillar and laryngeal 4; pharyngeal and laryngeal 2; total 127; number of deaths 5 .

The ages were: Under 1 year 4 cases; between 1 and 5 years 55 cases; between 5 and 10 years 30 cases; between 10 and 20 years 13 cases; between 20 and 40 years 25 cases. Nearly ó0 per cent. occurred below the age of 5 years and about 25 per cent. between the ages of 5 and 10 years.

1. The deaths occurred below the age of 8 years and were as follows: Age 1 year, 7 months; variety, laryngeal and tonsillar; operation, intubation; time of injection, about seventy-two hours after injection; time of death, forty-nine hours after injection and intubation; cause of death, sepsis.

2. Age, 2 years, 2 months; variety, laryngeal, ton. sils, nasal, pharynx; operation, intubation; time of injection, about the fifth day; time of death, thirtythree hours after injection and intubation; cause of death, sepsis and nephritis.

3. Age, 1 year; variety, tonsils, nose, pharynx, lymphatics; time of injection about the fifth day; time of death, three days after injection; cause of death heart failure-a septic case.

4. Age, 8 years; variety, tonsils, nose, pharynx and larynx; time of injection, about the fifth day; time of death, nine days after injection; cause of death, heart failure.

j. Age, 1 year 1 month; variety, tonsils, nose, pharynx and larynx; time of injection, fourth day; time of death, five days after injection; cause of death, heart failure.

They were as regards sex, 51 males, 76 females.
The time in which injections were made: first day, 21 cases; second day, 52 cases; third day, 34 cases with one death; fourth day, 8 cases with one death; fifth day 6 cases with three deaths; sixth day, 3 cases; seventh day, 1 case; eleventh day, 1 case; seventeenth day, 1 case.

Many cases were immunized, using for that purpose antitoxin from several laboratories. In those cases immunized no visual traces of the disease were seen, although the bacteriologic examination frequently revealed the Löfler bacilli. In one case treated for diphtheria of the faucial variety (case 87) a re-infection took place on exposure six months afterward (case 123) the disease manifesting itself in the same way. This proves, that while antitoxin has curative and immunizing virtue, it does not confer lasting or permanent immunity; but the susceptibility to infection remains as in any other form of treatment.

The complications noted: Broncho-pneumonia five cases, only seen by me in the laryngeal variety. Nephritis one case. Albumin was found frequently both before and after injection, antitoxin not increasing the amount. Sepsis four cases. Eruptions noted in about fifteen cases, and was also noticed in several cases that received an immunizing dose.

Sequelæ: Undoubted paralysis of the lower extremities in a female suffering from diphtheria of the laryngeal variety with membranes on the tonsils, complicated broncho-pneumonia, intubated, the tube being worn twelve days, and who received 1,000 units of Behring's antitoxin. The age of this child was 1 year 10 months; the injection was made on the third day, and there was perfect recovery months afterward. Heart failure in two cases, after disappearance of the membranes; in one case three days after the visits ceased. Ozena was noticed in one case. Method of using the diphtheria antitoxic serum: Antitoxin should be chosen of undoubted reliability. I am pursuaded that my results are mainly due, not only to my method, but to the purity and efficacy of the antitoxin used The technique pursued in the one case is that pursued in all.

Antitoxin is graded as regards strength into what is termed by its originators antitoxic normals or immunity units, which is an amount of antitoxic serum required to save a 500 gramme guinea pig from a minimum fatal dose of the diphtheritic toxin. The number of immunity units per c.cm. gives a standard strength of the serum; for instance: one onehundredth c.cm. will protect a 500 gramme guinea pig, therefore $1 \mathrm{c.cm}$. of antitoxic serum, which protects 50,000 grammes of guinea pig, contains 100 immunity units, and would protect an individual weighing one hundred times as much as a guinea pig (about one hundred and twenty pounds, if the susceptibility were the same. The serum, of which $1 \mathrm{c.cm}$. equals 100 immunity units, is that with which most of my work was done. Serum of greater strength was used. Knowing the strength of the serum makes me have no regard for the quantity used, as I grade my dosage in immunity units and so apply it.

The technique. The antitoxin serum is used as a curative or immunizing agent by subcutaneous injection into the tissues of the body. For the purpose of making these injections any hypodermic syringe may be used if of sufficient capacity, as the Pravaes syringe, the Koch bulb syringe; I prefer a special antitoxin syringe, made for this purpose, having a capacity 
of 10 c.cm., and which can be measured accurately by a screw, so that the quantity used can be administered in one injection. This syringe is supplied in metal case, with vulcanized rubber packing, thus permitting complete sterilization of both syringe and case. I have discarded asbestos packings since they become soft and pulpy.

The parts chosen by myself are in the back between the scapulæ on either side of the vertebral column. Other parts of the body, as the loins, groins or sides of the chest have been selected.

The parts are thoroughly cleansed by means of alcohol soaked upon sublimate cotton or gauze, and after injection are hermetically sealed with iodoform collodion.

The syringe is cleansed by means of very hot water and the whole operation rendered as aseptic as possible. I have yet to record an abscess, or even any inflammatory reaction following at the seat of injection, all of which $I$ ascribe to the rigid cleanliness enforced, in the use of my needles as well as in the parts anted upon.

The dosage. The amount of antitoxin used depends upon the time of making the injection, the age and body weight of the individual, and the gravity of the disease, also whether for the purpose of immunization or cure.

Experience has taught me that the method arrived at by the originators of the antitoxin as to the quantity of serum necessary to antagonize the toxins of diph theria was the correct one; I therefore follow the original method and grade my dose into immunity units.

As an immunizing dose I give an injection of 100 units to perfectly healthy individuals exposed to the contagion-their body weight averaging 120 pounds, basing the dose on these being of equal susceptibility to the guinea pig. This dosage is now the general rule. If there be constant exposure I increase this dosage to 200 units. By constant exposure I mean where the individual to be protected lives in the same room or house, as the tenement system of any large city. In extraordinary instances, as a woman in the last days of pregnancy. about to be confined, I inject a full dose, 600 units, as an immunizing dose. Of such cases I have seen two instances with the most satisfactory results; the accouchement taking place in one instance in the very room occupied by the still affected child, and no symptoms being manifest.

As a curctive dose. If the case be seen early, within twenty-four to forty-eight hours, and is of the faucial variety, where the infection has not reached the lymphatics, I administer at once 600 units. If the case be seen on the third day, or if at the very beginning the infection is such that leads one to believe in the necessity of prompt heroic treatment, as in the laryngeal cases or where the lymphatics are involved, or where the bacteriologic examinations show mixed infections (streptococcus, staphylococcus, etc.), I immediately inject 1,000 units. I then await results. If in six to twelve hours no change takes place or the symptoms are aggravated, I again inject 1,000 or 1,500 units of the serum, and so on in increasing quantities until there be an amelioration of the symptoms shown by a decline in the pulse rate and temperature and an improvement in the general condition.

The quantity of antitoxin can be increased to enormous dosage; being harmless, no danger can be apprebended. I have used as high as 13,000 units in one case. Others have used 200 c.cm. $-20,000$ units, with complete recovery.

\section{Clinical manifestation of the diphtheria antitoxin} serum.

1. The effects on the pulse and circulation. In faucial diphtheria, within a very short period, about eight hours in the most favorable cases, the pulse rate declines and remains down and the circulation assumes the normal. This, however, may not be permanent, and if the rate increases again it is an indication for the administration of more antitoxin. Especially is this an indication in those cases where the toxemia is so marked that the temperature is only slightly above the normal or is subnormal. In laryngeal cases, however, the pulse rate remains high in the majority of cases, and this is seen especially in those cases intubated. The indication then is not so much for the antitoxin as for judicious collateral medication.

2 . Effects on the temperature. In the most favorable cases, by which I mean the pure and simple diphtheria, there is a rapid decline from any elevation of temperature to the normal, and this decline is permanent. Where, however, there may be a recurrence of the fever and no manifestation of any intercurrent complication as pneumonia, and the like, it is an indication for an additional administration of the antitoxin, even if the occurrence should take place in one, two or three days after the injection. An elevation of the temperature later than four days indicates a broncho-pneumonia or some other disease and should be treated accordingly. In laryngeal cases is this especially marked, and in those cases intubated it has been seen by myself even after the tube was withdrawn.

3 . Action on the diphtheritic membrane. The action of the antitoxin is visible to the naked eye by the effects on the diphtheritic membrane.

It limits the extension within twenty-four hours and promotes the separation in forty-eight to seventytwo hours.

As an indication for the sufficient use of the serum I have noticed a distinct red line surrounding the membrane, making a line of demarkation between the healthy and diseased mucous membrane. Over this line I have never seen the membrane spread, and when it has formed my experience has taught me to expect a very favorable prognosis in that especial case.

The membranes separate differently; in some cases it comes away in one piece, leaving a healthy mucous membrane beneath. In other cases, especially those in which there is mixed infection the membranes soften and become pultaceous, and seem to dissolve or melt away.

4. Effects in laryngeal diphtheria. When used early enough it prevents the spread of the membranes, thereby averting asphyxia, and avoids the operation of intubation or tracheotomy. The membranes disappear in the same manner as in the faucial variety, stenosis being invariably relieved on the third day. Where the laryngeal diphtheria is complicated with membrancs on the tonsils, pharynx or nose, these disappear at the same time as the stenosis.

Intubated cases. Of the forty-four cases of laryngeal diphtheria, sixteen cases necessitated the operation of intubation, three of which were within the twenty-four hours after injection: the others were intubated before or at the time of the injection.

The average reduction in the time the tube was 
worn was sixty-nine hours. Previous to the serum period the average time was 185.25 ; since this period the average time was 116.25 hours. This reduction was noticed and soon recorded by Huebner (Klinische Studien, etc.), Von Ranke, Johan Bokai, O'Dwyer and others.

Inasmuch as the indication for tracheotomy was the length of time the tube was worn, with the concurrent symptoms, decubitus, etc, the time being placed above 120 hours. No urgent symptoms were manifested even in the long cases to necessitate tracheotomy. For that reason antitoxin has proven itself of the utmost value and is one of the conspicuous evidences of its effects.

Antitoxin has reduced the mortality in cases of intubation. In my cases so treated the reduction has been from a mortality of 62 per cent. before the serum period, to 10.5 per cent with antitoxin. Nor do I stand alone in this experience. Bokai (Stephanie, Kinder hospital, Buda-Pesth) reports in his hospital work an increase of 17 per cent. in his cures. The reduction in the time the tube was worn in those cases of recovery was eighteen hours, the average being sixty-one hours serum period, against seventy-nine hours before the serum period.

Jos. O'Dwyer of New York, whose work on this especial method of treatment is so well known to you all, has very kindly sent me a short report of his cases, a transcription of which I give here:

"My results up to the present time in 500 cases, in a series of hundreds: First hundred-largely experimental-seventeen recoveries; second hundred, twenty-seven recoveries: third hundred, thirty recoveries: fourth hundred, twenty-six recoveries; fifth hundred, thirty-nine recoveries.

"The marked increase in the last series was due to the antitoxin, in thirty cases, of which there were twenty recoveries. In the seventy preceding cases in which no antitoxin was used, there were only nineteen recoveries, which was about the same percentage as in all the other series."

O'Dwyer's statistics substantiate my own; from a mortality ranging from 83 to 70 per cent. before the serum period his reduction has been to 30 per cent. The average time the tube was worn, serum period, was 80 hours; before this period the time was 147 hours. So the reduction in time in O'Dwyer's cases was 67 hours.

Its action on the duration of the disease. Antitoxin limits the duration of the disease to four or five days and shortens the period, which may reach to weeks.

When administered early in simple diphtheria all visual traces of the disease disappear on the third day.

In mixed contagion the diphtheria infection is antagonized and the complications treated without regard to the existence of diphtheria.

In the laryngeal variety the stenosis disappears the third day in those cases not requiring operative interference. In cases intubated, the tube can be withdrawn with a certainty and exactness on the fourth or fifth day, such a rule being unknown before the serum period.

The general condition of the patient in the majority of cases improves at once, and by its early use, even before depression was manifested; thereby preventing complications and sequelæ, the termination of which, even with the antitoxin treatment can not be averted.
As a prevention of the meddlesome and dangerous method of constant local and systemic treatment in vogue. As antitoxin acts specifically and the general condition of the patients improve so quickly, no necessity arises for the constant and persistent methods of applying local applications formerly pursued.

It is true that the membranes of the throat are the foci for the invasion of the system by the diphtheria toxin; it is equally true that the injection of the antitoxin antagonizes and prevents further infection. If this be so, and thousands of cases have clinically demonstrated it, the persistent endeavors to remove the membrane by hourly or half-hourly applications are not only superfluous but dangerous, as not only tending to keep the patient awake and in dread, but by weakening the recuperative powers open a way for complications and a dangerous or perhaps fatal sequelæ (paralysis, heart failure and the like).

For this reason when treating a patient with antitoxin, local applications may be made once in four to six hours, and simply for the purpose of cleanliness, or for the specific purpose of ridding the throat of the bacteria, thus preventing the danger of contagion to others.

Poisonous drugs, as corrosive sublimate and the like, are unnecessary, or if administered as indications might require (as ulcerations resulting from the diphtheria, etc., in long cases) may be given at longer intervals.

Nourishment and stimulants are required, but for shorter periods than before, two or three days, and dispensed with when no longer indicated.

While antitoxin is a specific for diphtheria, it is not a "cure all," and complications must receive thorough attention as if diphtheria no longer existed. If the circulation be weak and the case urgent, stimulants, strychnin, digitalis, camphor or ammonia may be indicated. Again if a complication, as pneumonia or nephritis, exists this should be treated without any regard to the diphtheria or intubation, by the wet packs or any method most favored by the operator. But the constant applications to the throat and syringing of the nose at too frequent intervals is to be deplored as tending to a fatal result.

On the presence of bacilli in the throat. Antitoxin does not destroy the Klebs-Löffler bacilli in the throat. They persist as long after convalescence as under any other form of treatment.

It is therefore well to make frequent cultures before permitting the patient to mingle with others, thereby spreading the contagion.

I am at present studying what remedies are most useful in destroying these bacilli, and $I$ have the hearty coöperation of Dr. B. Meade Bolton, the direc. tor of the bacteriologic laboratory in Philadelphia, who examines these cultures for me.

I have used calomel combined with salol; corrosive sublimate, Löffler's solutions and a solution of nascent chlorin with tincture of the chlorid of iron, which I have used for a long period. The Löffler solutions and the chlorin iron mixture have given me the best results.

The formulæ of the Löfller solutions are as follows: No. 1.

Menthol. . . . . . . . . . . 10 grms.

Toluol, q. s. . . . . . . 36 c.c

Alcohol absol. . . . . . . . . . 60 c.c

Liq. ferri sesquichlor . . . . . . 4 c.c. 
No. 2.

Menthol.

Toluol, q. s.

Creolin.

Alcohol absol.

10 grms

36 c.c.

2 c.c.

65 c.c.

They are used by applying locally to the throat or nose by a mop or spray. Löfller's No. 1 is a painful application and is very much objected to for that reason, and can not be frequently used. The No. 2 is less painful and is applicable to a wider range of use.

The chlorin-iron mixture is administered in teaspoonful doses to a child 5 years of age once in two or three hours, during the day time only.

The formula is:

Potassii chloridi

Ac. hydrochloric, C. P.

Tinct. ferric chlor.

Syr. simplicis or glycerini

$\bar{a} \bar{a} \quad 100$

5,00

120,00

The bacilli disappeared under either of these medicaments in two weeks.

Guaiacol has been used by Dr. Martin W. Barr and Dr. Solomon Solis Cohen as a local application. The application is as painful as Löffler's, though Dr. Cohen has obviated this somewhat by the addition of menthol. Their results have been good, though systematic bacteriologic examinations have not demonstrated any superiority over my methods.

As an immunizing agent. That antitoxin produces immunity against an attack of diphtheria has been abundantly verified by a vast number of observers both in hospital experience and in private practice.

The quarantine system in vogue in Philadelphia has given me frequent opportunities to verify the assertion of Behring, Roux and others. This immun. ity is not lasting. Biggs of New York places it (from careful observation) at thirty days, Behring places it from six to eight weeks. I have seen no case immunized show clinical traces of the disease, though frequently Löffler's bacilli were found in the throat; where such cases existed, they were carefully watched and cultures made from time to time (once in three days). No other treatment except the immunizing dose was used, and cultures made and examined by Dr. B. Meade Bolton or Dr. H. D. Pease evinced the the presence. of the bacilli even to three weeks, though no visual traces were ever seen.

Antitoxin does not confer permanent or lasting immunity from diphtheria even to those who suffered from an attack of diphtheria and were treated with a curative quantity of the serum. For the susceptibility to a reinfection on exposure still remains with the patient, and therefore it should always be proper to immunize the patient whenever exposed.

The influence of antitoxin on the mortality records. There is no city in the world whose records have been carefully kept that has not shown a marked reduction in the death rates by the antitoxin treatment. might except Philadelphia, which seems to be behindhand in the method of presenting her records as compared with other cities.

There is no hospital in the world where antitoxin has been used that has not shown a reduction in the death rate. Again I must note exception to this by the records of the Philadelphia Municipal Hospital, where it appears only hopeless or the most serious cases are sent, for this hospital holds the most unique position of recording an increased mortality from this method of treatment.

There is hardly an exception to be noted in the statistics of private practitioners, which also show a reduction in the death rate.

Still, despite these positive proofs of the utility of the serum treatment; there seems to be a passive antagonism by some to this form of treatment. I can only explain it in the words of Dr. Charles L. Dana, New York (Diphtheritic Palsies and the Use of Antitoxin, Medical Record, April 11, 1896), from whose excellent monograph I quote: "While the treatment of diphtheria by antitoxin is a method that has been approved in all parts of the world and is upheld by a volume of statistics whose brute force is almost everwhelming, yet there is still some degree of skepticism about its real value. Most of the skepticism is the result of profound and banal ignorance, coupled, perhaps, with some hysterical obsession in the lines of zoöphilism and antivivisection. There are, however, some conservative physicians whose character and attainments we respect, who still hold a position of reserve, if not of disbelief, as to the value of the method, and they advance some very ingenious explanations of the way in which the statistics that so apparently favor antitoxin are gotten up."

These statistics are supposed to contain in a majority of instances cases whose recognition is simply bacteriologic and where the clinical diagnosis is secondary. Even if this be so, and I for my part have not counted as cases treated those immunized in whom the specific bacilli were found. I think the statistics are superior to the former for this reason: Given a case of diphtheria as formerly treated prognosis was out of the question; a mild case might suddenly become a serious one. A laryngeal case may become tracheal. With antitoxin the disease is aborted and the serious lesions now so commonly noted by different observers as broncho-pneumonia, multiple neuritis, post-diphtheritic palsies, etc., were not noted before because the patients perished long before these complications were noted. Therefore, given a case as now treated with antitoxin, if the serum is used early enough and in a judicions manner, mortality records should be simply to prove exceptions to the rule, and the death rate noted show a lack of careful observance of the earliest symptoms of diphtheria.

\section{AN INTRODUCTION TO THE DISCUS- SION UPON "BLOOD-SERUM THERA- PEUTICS."}

Read in the Section on Materia Medica, Pharmacy and Therapeutics a the Forty-seventh Annual Meeting of the American Medical Association at Atlanta. Georgia, May 5.8, 1896.

BY HAROLD C. ERNST, A.M., M.D. PROFESSOR OF BACTERIOLOGY IN HARVARD UNIVERSITY.

Probably the most radical change in the ideas of the causation of disease that has ever occurred has taken place during the last thirty years, its final outcome being the subject of the discussion before this meeting to-day. The steps leading up to the position at present occupied have been taken only after many wanderings and painful experiences, but as a result we seem to have reached a rational standpoint from which further progress may be made. The basis of blood-serum therapeutics is the idea of immunity, and the procedure is the result of the investigations made to determine how immunity occurs. It had been known for a long time that after attacks of certain

1 In the preparation of this paper I bave received much assistance from, the little work of Lachalme," Blood-Serum Therapeutics, P'aris, 